\title{
In Situ TEM Observation of Domain Behavior in Multiferroic Structures Under Applied DC Bias
}

\author{
C.R. Winkler, ${ }^{*}$ L.W. Martin, ${ }^{* *}$ C.L. Johnson, ${ }^{*}$ and M.L. Taheri* \\ * Department of Materials Science \& Engineering, Drexel University, Philadelphia, PA 19104 \\ ** Materials Science Division, Lawrence Berkeley National Laboratory, Berkeley, Berkeley, \\ California 94720
}

Select multiferroic materials exhibit ferroelectric and magnetic order, and the two order parameters are coupled through a quantum-mechanical exchange interaction [1,2]. One of the most widely studied magneto-electric multiferroics is the perovskite $\mathrm{BiFeO}_{3}(\mathrm{BFO})$. The magneto-electric coupling in BFO allows control of the ferroelectric and magnetic domain structures via applied electric fields. Furthermore, BFO films possess high Néel and Curie temperatures and large ferroelectric polarization. Recent advances in synthesis techniques have enabled the growth of highquality, epitaxial thin films [1]. Because of these unique properties, BFO and other magneto-electric multiferroics constitute a promising class of materials for incorporation into devices such as highdensity ferroelectric and magnetoresistive memories, spin valves, and magnetic field sensors $[3,4]$.

Before BFO can be integrated into devices, however, a full understanding of its ferroelectric and antiferromagnetic domain behavior across a range of time and length scales needs to be developed [1]. Improved control of ferroelectric domain structures is critical for increasing the performance of ferroelectric and magnetoresistive memories, because memory switching speed and capacity are limited by domain wall mobility and domain size, respectively.

We investigated the ferroelectric domains in BFO using diffraction contrast transmission electron microscopy (TEM) and high-resolution electron microscopy (HREM). Diffraction contrast was used to distinguish adjacent domains with different polarization directions (Fig. 1A), and HREM images were analyzed to determine the atomic structure of domain walls (Figs. 1B and 1C). We will present in situ TEM experiments using our custom-built biasing holder provided by Hummingbird

Scientific $^{\mathrm{TM}}$. The holder was designed to probe the response of BFO thin films to a DC bias applied through a set of epitaxial in-plane electrodes, thereby enabling control of ferroelectric switching in the BFO thin film. Domain wall movement will be captured using digital streaming video at $50 \mathrm{~Hz}$, at both low and high magnifications, and this information will be analyzed to measure domain kinetics and nucleation energies.

Correlating the results of the biasing experiments with domain morphology will lead to improvements in quantitative models that describe and predict domain kinetics across a range of length scales from micrometers to nanometers. These improved models will facilitate development of permanent memory chips with higher speeds and greater densities than those of the current generation [5]. 
References

[1] R. Ramesh \& N.A. Spaldin, Nature Mater. 6 (1), (2007) 21.

[2] C. Ederer \& N.A. Spaldin, Curr. Opin. Solid State Mater. Sci. 9 (3), (2005) 128.

[3] Y.H. Chu et al., Nature Mater. 7 (8), (2008) 678.

[4] N.A. Spaldin \& M. Fiebig, Science 309 (5733), (2005) 391.

[5] This work was supported by the National Science Foundation and Drexel University through the GAANN-DREAM fellowship under contract P200A060117. Use of the Department of Energy facilities at the Center for Integrated Nanotechnology at Sandia National Laboratories is gratefully acknowledged. We thank Dr. Lolita Rotkina of the University of Pennsylvania and Asher Leff of Drexel University for assistance with sample preparation.
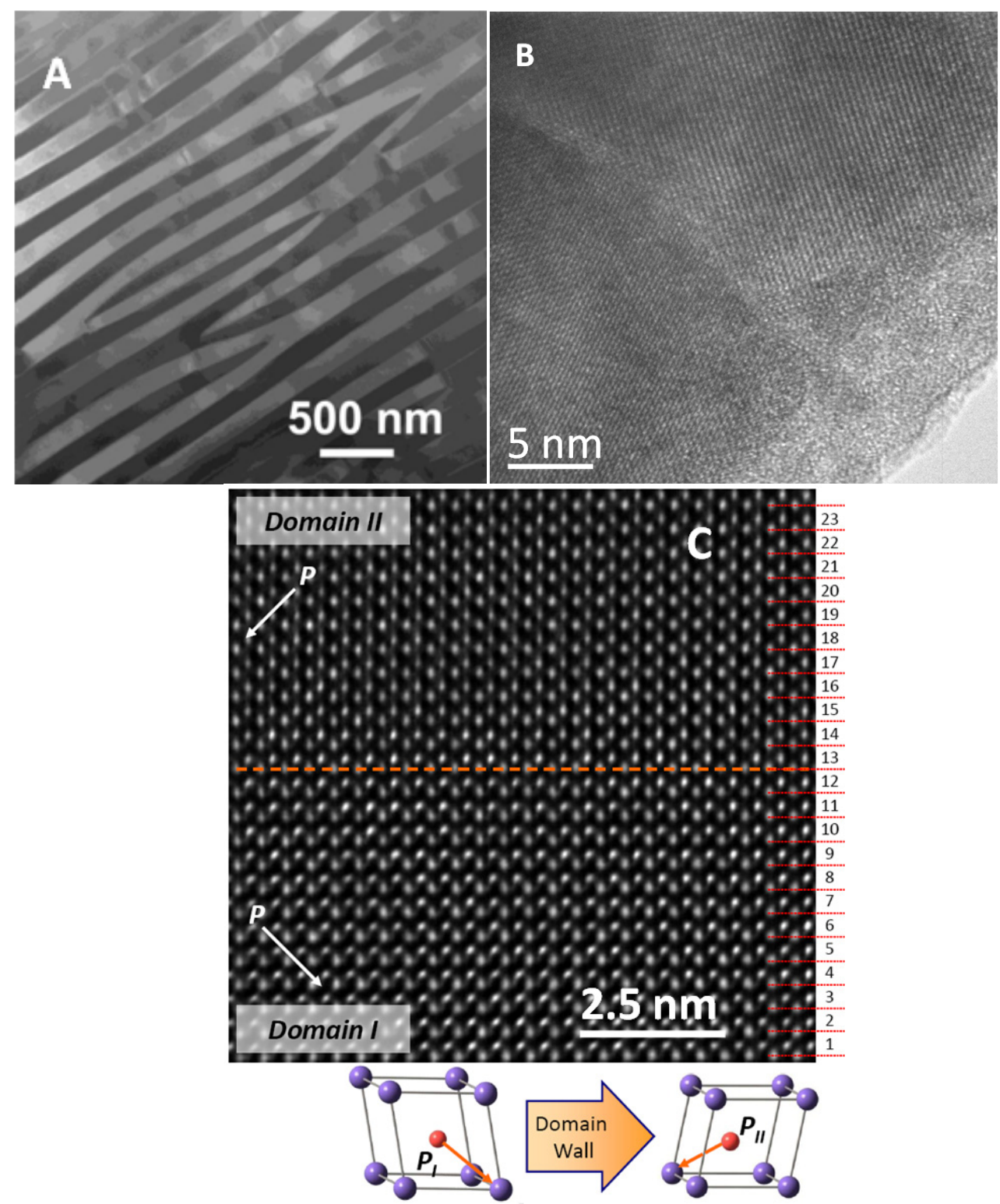

FIG. 1. TEM analyses of domain structures in BFO thin films. A) The diffraction-contrast TEM image shows striped domains in BFO thin films. B) The HREM image shows a domain wall in a BFO thin film. C) The exit-wave-reconstructed HREM image shows the boundary between two differently polarized ferroelectric domains, which are depicted by a schematic diagram. 